\title{
Primeros indicios de reproducción y amplia- ción de registros de Tarentola mauritanica (Linnaeus, 1758) en Álava (País Vasco, España).
}

First signs of reproduction and the increase in records of Tarentola mauritanica (Linnaeus, 1758) in Álava (Basque Country, Spain).

Conrado Tejado-Lanseros $^{1 *}$, María Elena Potes ${ }^{1}$

*

\section{Resumen}

La salamanquesa común, Tarentola mauritanica (L., 1758) cuenta con registros en el centro y norte de Álava desde el año 2006, en la presente nota se incrementa el número de observaciones comprendidas dentro del periodo 2011-2021. En esta década se observan por primera vez ejemplares neonatos y juveniles. Se valora la existencia de una población incipiente en determinados núcleos urbanos y rurales de la Llanada Alavesa.

Palabras clave: Tarentola mauritanica, neonatos, juveniles, Álava, País Vasco.

\section{Abstract}

There have been records of the common gecko, Tarentola mauritanica (L., 1758) in the centre and north of Alava since 2006. In this note, the number of observations increased during the period 2011-2021, with citations of adult, juvenile and neonatal specimens. The existence of an incipient population in certain urban and rural areas of the plain of Alava is assessed.

Key words: Tarentola mauritanica, neonates, juveniles, Álava, Basque Country.

1 Instituto Alavés de la Naturaleza.

Departamento de Zoología-Vertebrados.

Apdo. 209201008 Vitoria-Gasteiz.

*Correspondencia: info@ian-ani.org; conradotejado@gmail.com 


\section{Laburpena}

Dragoitxo arruntaren, Tarentola mauritanica (L., 1758) Araba erdialde eta iparraldeko aipuak bildu izan dira 2006 urtetik hona, eta idatzi honetan 2011-2021 urteen arteko aipu kopurua gehitu egin da. Hamarkada honetan bildu diren behaketetan, lehen aldiz neonatoak eta gazteak daude. Populazio hasberrien presentzia begiesten da Arabako lautadako gune urbano eta landatarrean.

Gako hitzak: Tarentola mauritanica, neonatoak, gazteak, Araba, Euskal Herria.

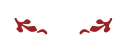

A lo largo de las dos últimas décadas se han venido recopilando registros de Tarentola mauritanica en la provincia de Álava y áreas limítrofes. En su mayor parte tienen como denominador común su carácter puntual, la ubicación en hábitats antrópicos y la observación de individuos aislados.

El análisis de la situación de la especie en la primera década del siglo XXI, entre los años 2006-2009, no confirmaba la existencia de poblaciones establecidas ni ejemplares juveniles, aunque preveía el incremento de su área distributiva y su posible estabilización en forma de pequeños núcleos reproductores (Tejado \& Potes, 2011, 2016).

Existen evidencias de la dispersión de este reptil termófilo hacia zonas más septentrionales de la península ibérica. Por ejemplo, la especie ha alcanzado puntos de la costa atlántica de Galicia (Piorno, Martínez \& Fernández, 2017) y la costa cantábrica en el País Vasco, donde además se han detectado ejemplares neonatos (Gosá et al., 2011).

Dentro del Territorio Histórico de Álava ha aumentado sensiblemente el número de citas en el periodo 2011 - 2021, según se puede establecer por comparación con registros anteriores al 2011 y, a diferencia de la década anterior, se han localizado individuos neonatos y juveniles (Fig.- 1, Tabla 1). La distribución de la especie en la región parece estar claramente condicionada por el hombre al situarse la totalidad de los avistamientos en núcleos urbanos (Vitoria-Gasteiz-Llodio) y rurales (Argómaniz, Santa Cruz de Campezo), fruto de su dispersión a larga distancia, de forma pasiva, por medios de transporte como camiones de mercancías o ferrocarril. Al respecto es necesario señalar la recogida de ejemplares adultos en el Centro de Recuperación de Especies Protegidas (C.R.E.P.) de Martioda, hallados en camiones de mercancías del polígono comercial de Etxabarri-lbiña en 2013 (Mikel Salvador, com. pers). Así como individuos transportados involuntariamente, ocultos entre material de escalada desde la Sierra del Montsec (Lleida) en 2019, que fueron liberados en su lugar de origen (Ricardo Gutiérrez com. pers.). El modo de dispersión accidental es particularmente aplicable a este gecónido que, favorecido por su habitual convivencia con el ser humano, ha sido introducido en buena parte de su distribución a nivel mundial (Rato, 2015). 


\begin{tabular}{|c|c|c|c|c|c|}
\hline Cita & Localidad & Fecha & $\begin{array}{c}\text { Coord. X/Y } \\
\text { (ETRS89) }\end{array}$ & $\mathrm{N}^{\circ}$ ej. & Enclave/Hábitat \\
\hline 1 & Vitoria & 15.09 .2011 & $526890 / 4743294$ & 1 Adulto & Pared patio urbano ajardinado \\
\hline 2 & Etxabarri-Ibiña & 18.01 .2013 & 523382 / 4750988 & 1 Adulto & Camión de mercancías \\
\hline 3 & Vitoria & 28.07 .2014 & $527260 / 4743852$ & 1 Adulto & Pared vivienda urbana \\
\hline 4 & Vitoria & 26.03 .2016 & $526890 / 4743294$ & 2 Adultos & Pared patio urbano ajardinado \\
\hline 5 & Llodio & 20.05 .2017 & 503257 / 4776661 & 1 Adulto & Muro de piedra \\
\hline 6 & $\begin{array}{c}\text { Sta. Cruz } \\
\text { de Campezo }\end{array}$ & 31.05 .2018 & 552882 / 4724348 & 1 Adulto & Pared vivienda rural \\
\hline 7 & Vitoria & 17.07 .2018 & $526139 / 4743515$ & 1 Adulto & Garaje edificio urbano \\
\hline 8 & Vitoria & 21.02 .2019 & $526086 / 4744009$ & 1 Neonato & Pared camarote urbano \\
\hline 9 & Vitoria & 12.07.2019 & $524615 / 4744883$ & 1 Adulto & Muro calle urbana \\
\hline 10 & Vitoria & 24.08 .2019 & $526600 / 4743348$ & 1 Adulto & Muro calle urbana \\
\hline 11 & Argómaniz & 20.09 .2019 & $537270 / 4746301$ & $\begin{array}{l}1 \text { Adulto + } \\
1 \text { Juvenil }\end{array}$ & Pared vivienda rural \\
\hline 12 & Vitoria & 26.10 .2019 & 524615 / 4744883 & 1 Adulto & Pared vivienda urbana \\
\hline 13 & Vitoria & 17.11 .2020 & 527425 / 4742781 & 1 Neonato & Pared patio urbano \\
\hline 14 & Vitoria & 12.04 .2021 & 525172 / 4745480 & 1 Adulto & Foso ascensor vivienda urbana \\
\hline 15 & Vitoria & 14.04 .2021 & 527747 / 4744955 & 1 Adulto & Pared vivienda urbana \\
\hline 16 & Argómaniz & 18.04 .2021 & 537444 / 4746063 & 1 Juvenil & Pared vivienda rural \\
\hline 17 & Vitoria & 24.06 .2021 & 525585 / 4744171 & 1 Adulto & Suelo calle urbana \\
\hline 18 & Vitoria & 07.08 .2021 & $526890 / 4743294$ & 2 Adultos & Pared patio urbano ajardinado \\
\hline 19 & Vitoria & 21.08 .2021 & 527366 / 4742676 & 1 Adulto & Pared vivienda urbana \\
\hline 20 & Vitoria & 03.09 .2021 & $526988 / 4745115$ & 1 Juvenil & Pared vivienda urbana \\
\hline 21 & Vitoria & 13.09 .2021 & 527179 / 4743290 & 1 Adulto & Local comercial urbano \\
\hline 22 & Vitoria & 21.09 .2021 & 526102 / 4745777 & 1 Joven & Parque urbano \\
\hline 23 & Vitoria & 27.09 .2021 & 528809 / 4746913 & 1 Juvenil & Muro calle urbana \\
\hline 24 & Vitoria & 05.10 .2021 & 525879 / 4745696 & 1 Adulto & Pared vivienda urbana \\
\hline
\end{tabular}

Tabla 1.- Observaciones de Tarentola mauritanica en Álava (2011-2021).

Table 1.- Records of Tarentola mauritanica in Álava (2011-2021).

Atendiendo al comportamiento estacional de la especie, en Álava se han realizado observaciones de ejemplares activos en todas las estaciones del año, según los registros cronológicos aportados en la Tabla 1, así como recepción de individuos en el C.R.E.P. de Mártioda, procedentes del entorno urbano de Vitoria-Gasteiz. Algunas de 


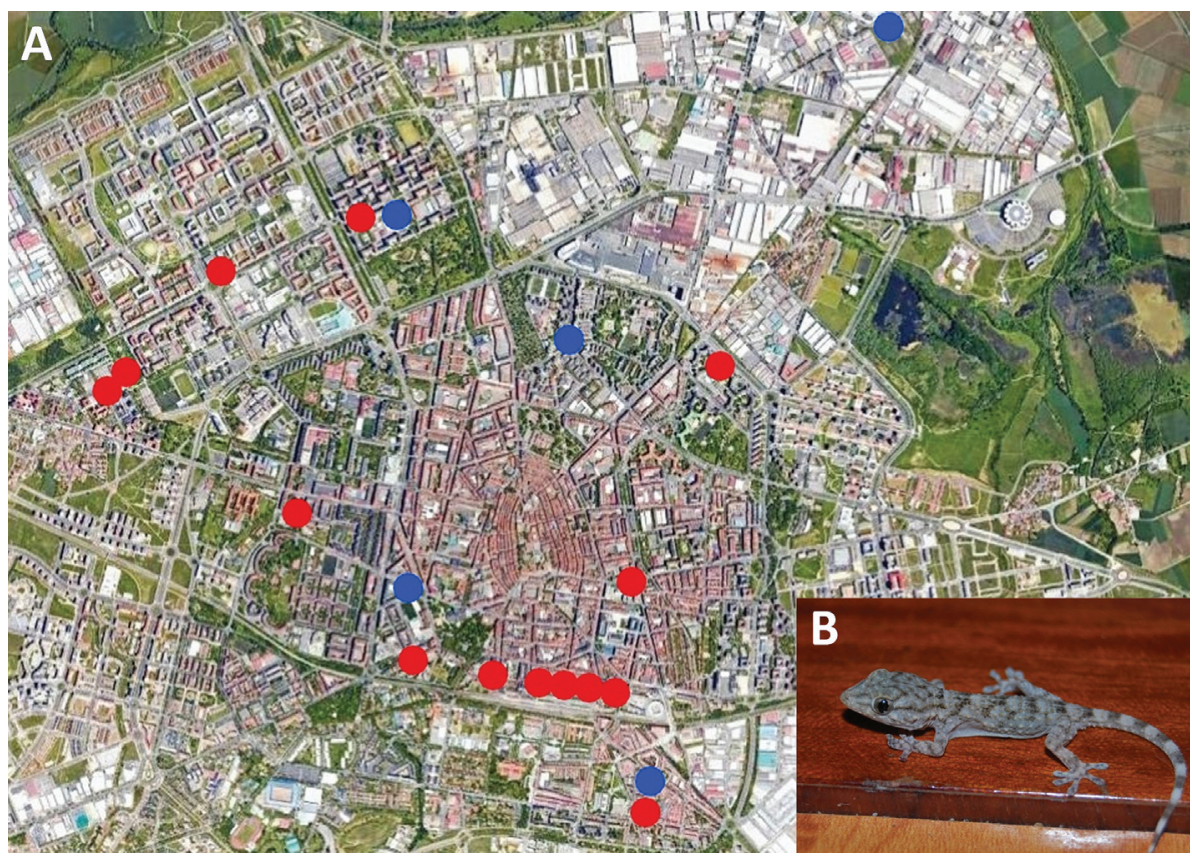

Fig. 1.- (A) Registros de Tarentola mauritanica en la ciudad de Vitoria-Gasteiz, España (2011-2021). Los puntos azules indican presencia de ejemplares neonatos y juveniles. (B) Neonato de Tarentola mauritanicade $59 \mathrm{~mm}$ (longitud total), fotografiado en el sur de Vitoria-Gasteiz (Cita número 13).

Fig. 1. - (A) Tarentola mauritanica records in Vitoria-Gasteiz, Spain (2011-2021). Blue dots indicate the presence of neonates or juveniles specimens. (B) Tarentola mauritanica newborn of $59 \mathrm{~mm}$ in overall length, photographed in the south of Vitoria-Gasteiz (Record number 13).

las observaciones de estos reptiles heliotérmicos, realizadas en mañanas frías de periodo otoñal, se localizan en muros orientados hacia el sur junto al trazado férreo. Se muestra una consonancia con estudios llevados a cabo en poblaciones de salamanquesas comunes introducidas en Galicia, donde T.mauritanica está activa en periodos anticiclónicos en pleno mes de diciembre (Galán, 2020). Igualmente, en otros puntos de la península Ibérica y Baleares, esta especie presenta actividad diurna en periodo invernal (Escarré \& Vericad, 1981; Busack \& Jaksic, 1982; Cerdeira \& Ribot, 2006).

Es destacable señalar que parte de las observaciones en Álava reportadas en este trabajo (2011-2011) corresponden a ejemplares neonatos y juveniles (Tabla 1), fenómeno desconocido hasta el momento en el área y no registrado en estudios precedentes (Zaldívar, 2000; Tejado \& Potes 2011). Las observaciones y capturas de individuos neonatos y juveniles en las localidades de Vitoria-Gasteiz y Argómaniz, junto a las citas reiteradas durante varios años en las mismas cuadrículas, (citas número 1, 4, 7, 10, $11,13,15$ y 18) constituyen los primeros signos de una población incipiente. Dos ejemplares capturados para la toma de datos biométricos en 2019 y 2020, dentro de 
la ciudad de Vitoria-Gasteiz, ofrecieron medidas corporales de $59 \mathrm{~mm}$ (longitud total) - $29 \mathrm{~mm}$ (hocico-cloaca [SVL]) y $50 \mathrm{~mm}$ (longitud total). Estas medidas se sitúan dentro del margen 40-58 mm (longitud total), establecido para los neonatos y juveniles del año (González de la Vega, 1988).

Los patrones de registros iniciales, el proceso cronológico de ocupación, y la reciente aparición de neonatos, se ajustan a las tendencias observadas en otras ciudades no costeras del noroeste e interior peninsular, con condiciones invernales tanto o más extremas que las de Vitoria-Gasteiz. Como son los casos de Orense y Soria, donde $T$. mauritanica se introdujo a partir de procesos de dispersión pasiva de carácter antropogénico (Cabana, 2008; Meijide et al., 1994). El índice pluviométrico que soporta la Llanada Alavesa tampoco puede considerarse un obstáculo, ya que parece que la especie es tolerante a estas condiciones ambientales lo que le ha permitido establecer poblaciones reproductoras bien asentadas en zonas de clima atlántico, con inviernos especialmente húmedos y lluviosos, como es la ciudad de A Coruña (Galán, 2018). Así como en países norteños (e. g. Holanda) muy alejados de su área de distribución original (Peek, 2012).

La observación de este conjunto de factores, junto a los registros de la última década, nos permite argumentar que es alta la probabilidad de que en las próximas décadas, esta especie llegue a desarrollar una población más numerosa y asentada en determinados núcleos de una comarca de clima subatlántico como es la Llanada Alavesa. La vinculación a núcleos urbanos y rurales con presencia de refugios seguros y escasez de enemigos naturales le permitiría mantener poblaciones emergentes en estos espacios humanizados.

\section{Agradecimientos}

A Anto Aguilar, Roberto Alcácer, Enrique Arberas, Nieves Díaz de Argote, Norber Fuente, Xana González, Ricardo Gutiérrez, Martín Rezola y Mikel Salvador por la aportación de observaciones y documentación fotográfica, sin cuya colaboración no hubiera sido posible la elaboración del presente artículo.

\section{Bibliografía}

Busack, S.D., Jaksic, F.M., 1982. Ecological and historical correlates of Iberian herpetofaunal diversity: an analysis at regional and local levels. J. Biogeogr. 9, 289-302.

Cabana, M., 2008. Nuevas citas de Tarentola mauritanica en Galicia: ¿especie alóctona o autóctona en la zona? Boletín Asociación Herpetológica Española 19, 59-60.

Cerdeira i Ribot, J. ,2006. Localización de Tarentola mauritanica (Linné, 1758) en cajas nido para pájaros: ¿Un indicador de elevada actividad arborícola estacional? Boletín Asociación Herpetológica Española 17(1), 38-41. 
Escarré, J., Vericad, J.R., 1981. Fauna alicantina. I.-Saurios y ofidios. Instituto Estudios Alicantinos, Alicante.

Galán, P., 2018. Presencia y uso del espacio de una población introducida de Tarentola mauritanica en el norte de Galicia. Boletín Asociación Herpetológica Española 29(1), 35-41.

Galán, P., 2020. Nueva introducción de Podarcis muralis en Galicia, en una zona ya invadida por Tarentola mauritanica: el transporte de madera como vehículo de especies invasoras. Bol. Asoc. Herpetol. Esp. 31(1), 82-85.

González de la Vega, J.P., 1988. Anfibios y reptiles de la provincia de Huelva. Ertisa, Huelva.

Gosá, A., Laza-Martínez, A., Crespo-Díaz, A., Sanz-Azkue, I., Valdeón, A., Rubio, X., 2011. Reproducción de Tarentola mauritanica (L., 1758) en la costa vasca. Munibe, Cienc. nat. 59, 95-101.

Meijide, M., Meijide-Fuentes, F., Arribas, O., 1994. Atlas herpetológico de la provincia de Soria. Rev. Esp. Herpetol. 8, 45-58.

Peek, R., 2012. Langdurige overleving van de Muurgekko (Tarentola mauritanica). Lacerta 70(1), 32-33.

Piorno, V., Martínez, L., Fernández, J.A., 2017. Un caso de dispersión a larga distancia de salamanquesa común mediada por el hombre. Bol. Asoc. Herpetol. Esp. 28 (1), 83-84.

Rato, C., 2015. La salamanquesa común (Tarentola mauritanica) en la Península Ibérica e Islas Baleares. Boletín Asociación herpetológica Española 26(2), 55-58.

Tejado, C., Potes, M.E., 2011. Primeros registros de Tarentola mauritanica (L., 1758) para el centro y norte de Álava. Munibe, Cienc. nat. 59, 87-93.

Tejado, C., Potes, M.E., 2016. Herpetofauna del Territorio Histórico de Álava. Diputación foral de Álava, Vitoria-Gasteiz.

\section{光}

Fecha de recepción/ Date of reception: 06/08/2021

Fecha de aceptación / Date of acceptance: 14/10/2021

Editor Asociado / Associate editor: María Torres-Sánchez 